\title{
Hyperfine Parameters for Muonium in Copper (I), Silver (I) and Cadmium Oxides
}

\author{
S. F. J. COX ${ }^{1,2, *}$, J. S. LORD ${ }^{1}$, S. P. COTTRELL ${ }^{1}$, H. V. ALBERTO ${ }^{3}$, \\ J. M. GIL ${ }^{3}$, J. PIROTO DUARTE ${ }^{3}$, R. C. VILÃO ${ }^{3}$, \\ A. KEREN ${ }^{4}$ and D. PRABHAKARAN ${ }^{5}$ \\ ${ }^{1}$ ISIS Facility, Rutherford Appleton Laboratory, Chilton, OX11 0QX, UK \\ ${ }^{2}$ Condensed Matter and Materials Physics, University College London, London, UK \\ ${ }^{3}$ Department of Physics, University of Coimbra, Coimbra, Portugal \\ ${ }^{4}$ Technion, Haifa, Israel \\ ${ }^{5}$ Department of Physics, University of Oxford, Oxford, UK
}

\begin{abstract}
Muonium centres in $\mathrm{Cu}_{2} \mathrm{O}, \mathrm{Ag}_{2} \mathrm{O}$ and $\mathrm{CdO}$ show hyperfine parameters spanning four orders of magnitude. They exemplify the three different categories of hydrogen defect centre in semiconducting and dielectric solids, with very different electronic structure and electrical activity, namely quasi-atomic (possibly deep acceptor), deep donor and shallow donor.
\end{abstract}

Key Words: hydrogen, muonium, oxides.

Following the success of muonium spectroscopy in modelling the structure and electrical activity of hydrogen defect centres in semiconductors, including the elemental group-IV and compound III-V semiconductors as well as the widergap II-VI materials, it is timely to apply the technique to oxides, as these materials are being reappraised and developed as novel electronic materials. The overall systematics show three distinct categories of defect centre, as described in an accompanying paper [1], and we present here the latest examples of each type to be discovered, in $\mathrm{Cu}_{2} \mathrm{O}, \mathrm{Ag}_{2} \mathrm{O}$ and $\mathrm{CdO}$. Our measurements were made using the EMU and DEVA instruments on the ISIS pulsed muon source.

For $\mathrm{Cu}_{2} \mathrm{O}$ and $\mathrm{Ag}_{2} \mathrm{O}$, the character of the centres is best displayed using the longitudinal-field techniques known as repolarization and level-crossing resonance, as in Figure 1. These involve measuring time-average muon polarization as the magnetic field (applied parallel to the polarization of the incoming muon beam) is scanned through regions where the electron, muon and nuclear spin states are mixed or perturbed by the hyperfine and superhyperfine interactions. For $\mathrm{Cu}_{2} \mathrm{O}$, the repolarization is monotonic, representing a simple decoupling of these interactions. The muon-electron hyperfine interaction is fitted from this

* Author for correspondence. 


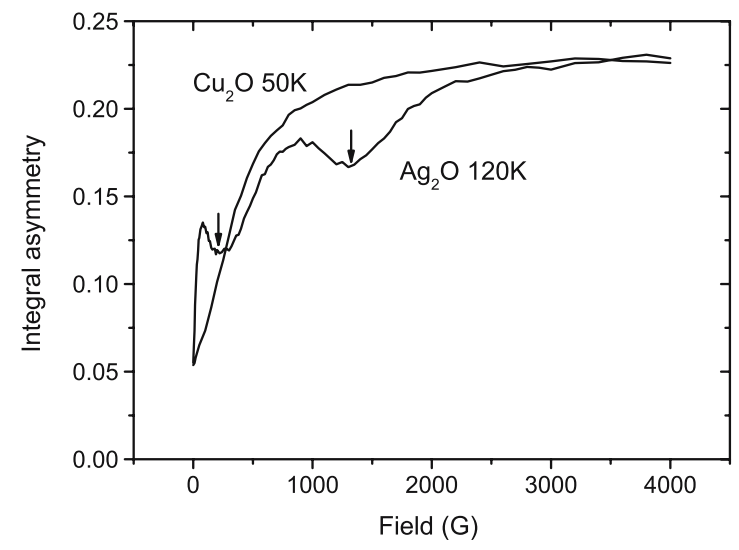

Figure 1. Repolarization curves for muonium in $\mathrm{Cu}_{2} \mathrm{O}$ and $\mathrm{Ag}_{2} \mathrm{O}$. The decoupling field for $\mathrm{Cu}_{2} \mathrm{O}$ is roughly one third that of atomic muonium; for $\mathrm{Ag}_{2} \mathrm{O}$ it is lower still, with level crossing resonances (arrows) defining the hyperfine parameters precisely.

curve as $1276 \pm 20 \mathrm{MHz}$, which is just $29 \%$ of the free-muonium value (4463 $\mathrm{MHz}$ ). This is amongst the lowest values for the trapped-atom or quasi-atomic defect centres but is close to those for muonium in the cuprous halides, which span $27 \%$ to $37 \%$. The low value suggests a considerable delocalization of spin density onto the surrounding ions, possibly involving transfer to the copper $3 \mathrm{~d}$ orbitals.

For $\mathrm{Ag}_{2} \mathrm{O}$, the steep initial repolarization indicates a much smaller muon hyperfine constant, but the curve is modified by the appearance of two striking resonances. The higher-field feature we take to represent a resonance of the type denoted $\Delta M=1$, in which the external field may be said to tune out parallel components of the hyperfine field, leaving the muon spin free to precess in transverse components [2]. The contact interaction is determined rather precisely as $37 \pm 0.2 \mathrm{MHz}$, though its sign is ambiguous: it may be that such a low value represents spin polarization of bonding orbitals. Detection of the resonance implies a degree of anisotropy that may be taken as evidence of directional bonding. The lower-field resonance could conceivably represent a second muonium centre with correspondingly smaller hyperfine constant. Alternatively, it is a resonance of type $\Delta M=0$ from the same centre. In this case, it represents a flip-flop exchange of polarization between the muon and neighbouring silver nuclei, mediated by their separate interactions with the unpaired electron.

Whereas the centre in $\mathrm{Cu}_{2} \mathrm{O}$ appears to be of the quasi-atomic type and is stable up to at least room temperature, that in $\mathrm{Ag}_{2} \mathrm{O}$ has hyperfine parameters reminiscent of bond-centred muonium in $\mathrm{Si}, \mathrm{Ge}, \mathrm{GaAs}$ and $\mathrm{GaP}$, where it behaves as a deep donor. Supporting this analogy, the repolarization and resonance features disappear between 150 and $250 \mathrm{~K}$ as the unpaired electron is lost by ionization. Figure 2 shows the growth of the muon Larmor precession signal in this régime as neutral muonium converts to the positive ion (the interstitial 

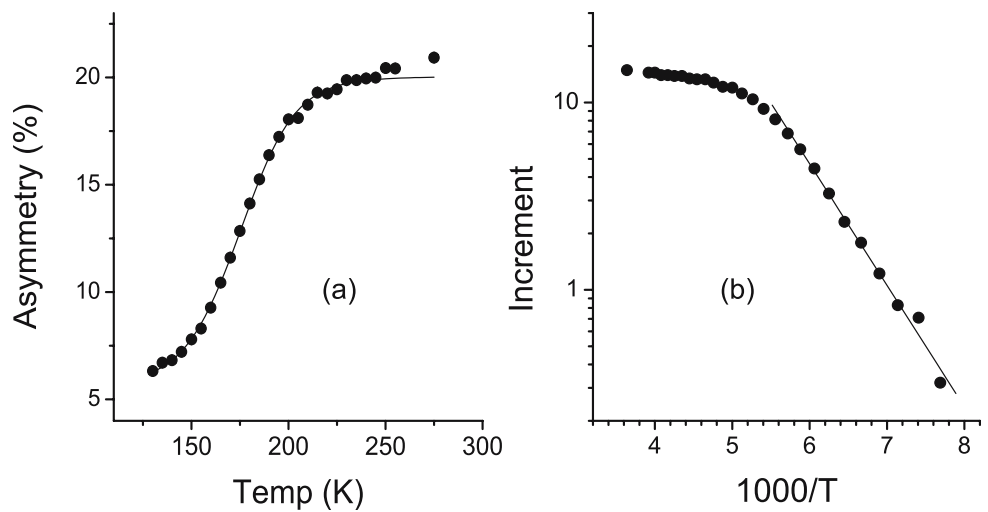

Figure 2. Variation of the diamagnetic or ionic fraction through the ionization régime, for muonium in $\mathrm{Ag}_{2} \mathrm{O}$. Fitted with an equilibrium model in (a), this yields a donor depth of $250 \mathrm{meV}$; an Arrhenius plot (b), implying direct loss of the electron to the conduction band, gives an ionization energy of half this value.

muon): this signal is measured by the more usual technique of muon spin rotation in transverse magnetic field. Analysis for the donor depth is model-dependent, but lies between 125 and $250 \mathrm{meV}$.

For $\mathrm{CdO}$, repolarization is complete at much lower fields and the hyperfine parameters are more conveniently determined from the muon spin rotation spectrum, recorded in transverse field. The low-temperature spectrum, obtained by a maximum-entropy transform of the time-domain signal, is shown in Figure 3(a). Satellite lines can be discerned, symmetrically placed about a larger central line. The satellites represent the small proportion of muons which pick up an electron to form paramagnetic muonium, with a hyperfine splitting of about 140 $\mathrm{kHz}$. The central line is the Larmor-precession frequency for muons which fail to pick up an electron on implantation, almost certainly thermalizing as the positive ion. This central line grows at the expense of the satellites in an ionization régime between 50 and $150 \mathrm{~K}$, as shown in Figure 3(b). The evolution is fitted here in the equilibrium model, to give a donor depth of about $90 \mathrm{meV}$.

In the shallow-donor model, the hydrogenic orbital is dilated both by the dielectric constant of the medium and by the low effective mass of the electron (the latter is weakly bound as a wave packet of conduction- band states). The effective mass is low (0.11) in $\mathrm{CdO}$ and the variation of dielectric constant unusually high $(\varepsilon(0)=21.9$ to $\varepsilon(\infty)=5.9)$. It is normal in effective-mass theory to use the low-frequency value but our results, both for contact interaction and ionization energy, only fit with this model if we use the high-frequency value of dielectric constant. This seems reasonable when ions as heavy as $\mathrm{Cd}$ are involved, although other possibilities must be considered, such as association of the muonium with a vacancy or other defect.

It seems likely that the quasi-atomic muonium centre in $\mathrm{Cu}_{2} \mathrm{O}$ represents the neutral state of a deep acceptor. However, the acceptor function itself, i.e., 

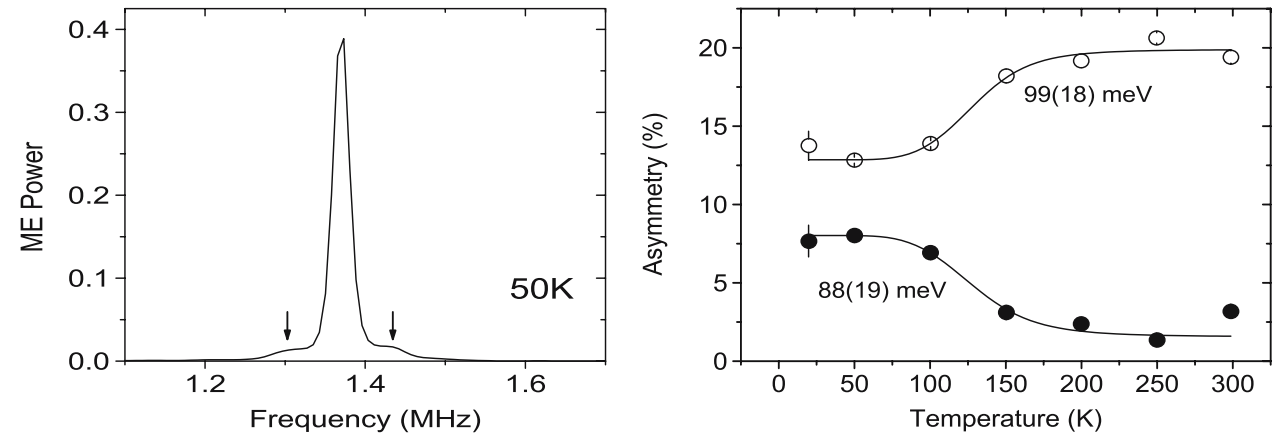

Figure 3. Muon spin rotation spectrum for $\mathrm{CdO}$ at $20 \mathrm{~K}$, with hyperfine satellites representing paramagnetic muonium (a) and analysis of the paramagnetic (lower) and diamagnetic (upper) amplitudes through the ionization régime (b).

conversion to the negative ion, whether by hole ionization or second electron capture, has yet to be demonstrated. The negative ion would be the muonium analogue of the hydride ion, whose importance, or even existence, in oxides is itself a matter of some topical interest. The muonium centre in $\mathrm{Ag}_{2} \mathrm{O}$ undoubtedly represents a deep donor whereas that in $\mathrm{CdO}$ is a reasonable candidate for an effective-mass shallow donor of the type established in $\mathrm{ZnO}$. (A similar contrast between deep and shallow character has been noted for muonium in $\mathrm{HgO}$ and $\mathrm{ZnO}$ [3].) The deep donor in $\mathrm{Ag}_{2} \mathrm{O}$ and the shallower centre in $\mathrm{CdO}$ are both fully ionized by room temperature so that, by implication, hydrogen impurity may act as an inadvertent dopant in these materials, inducing n-type conductivity.

\section{References}

1. Cox S. F. J. and Johnson C., (this issue, plus refs therein).

2. Roduner E., in Muon Science (Lee S. L., Kilcoyne S. H. and Cywinski R., eds), Scottish Summer Schools in Physics, vol. 51 (I.o.P. Bristol, 1998) p. 173.

3. Cox S. F. J., Davis E. A., King P. J. C., Gil J. M. et al., J. Phys. Cond. Matter 13 (2001), 9000 (plus references therein): Gil J. M. et al., ibid, L613.

Note added in proof. Both resonances for $\mathrm{Ag}_{2} \mathrm{O}$ in Figure 1 have since been established as having $\Delta M=1$ character: the low-field resonance is now assigned to a distinct muonium state with shallow-donor character, coexisting with the deep state responsible for the higher-field resonance. 